\title{
Univ.-Prof. Dr. Robert N. Braun (1914-2007)
}

21 Jahre nach der Publikation des „Lehrbuch der Allgemeinmedizin" (Theorie, Fachsprache, Praxis) erschien im Frühjahr 2007 eine Neuauflage, editiert von Braun, Fink. Am 13. September desselben Jahres starb Robert N. Braun im Alter von 93 Jahren. Der Autor dieser Zeilen kannte ihn seit vielen Jahren und lernte ihn als einen Freund kennen, von dem er - selbst schon Jahrzehnte auf medizinischem Gebiet tätig - aufgrund seines alles kritisch hinterfragenden und daraus entsprechende Konsequenzen ziehenden Geistes viele neue Einblicke in die Praxis der Medizin gewann. Dies schlug sich unter anderem auch in einer mehrjährigen Kooperation hinsichtlich wissenschaftlicher Publikationen in der Wiener Medizinischen Wochenschrift nieder.

Brauns Leben war ein Leben für eine lehrbare Allgemeinmedizin, für eine auf wissenschaftlichen Grundlagen basierende Ausübung der ärztlichen Tätigkeit besonders unter den Bedingungen des Praxisalltages.

Ursprünglich wollte er nach dem Abschluss seines Medizinstudiums im Jahre 1937 eine Universitätslaufbahn einschlagen, wurde aber von der 2. Medizinischen Universitätsklinik im Allgemeinen Krankenhaus Wien an die Front berufen. Im letzten Kriegsjahr war er in der deutschen Kleinstadt Marburg an der Lahn als praktischer Arzt dienstverpflichtet gewesen.

Das Resultat dieser ersten Erfahrungen in der allgemeinärztlichen Praxis war ein unveröffentlicht gebliebenes Buch mit dem Titel „Kritik am Arzttum und dessen Reform", das er 1945/46 geschrieben hatte. Er hatte bereits erkannt, dass in der Ärzteausbildung deutliche Mängel für eine spätere Tätigkeit als Allgemeinmediziner vorhanden waren, und auch dass dafür die theoretischen Grundlagen fehlten.

Nach dem Zweiten Weltkrieg praktizierte er zuerst in Wiener Neustadt und ab 1951 in einer kleinen Landpraxis in Brunn an der Wild im Waldviertel. Hier hatte er die nötige Zeit, um sich auch der Wissenschaft der angewandten Medizin, die er als Terra incognita beschrieb, zu widmen: Er setzte seine umfangreichen Praxisbeobachtungen fort, begann sie $\mathrm{zu}$ analysieren und $\mathrm{zu}$ veröffentlichen.

Im Jahre 1955 hielt er in der Gesellschaft der Ärzte in Wien einen Vortrag unter dem Titel „Über fundamental wichtige bisher unbekannte die allgemeine Morbidität betreffende Gesetzmäßigkeiten“. In Personalunion als Praktiker, Forscher und später auch Lehrer verfasste er im Laufe seines langen Lebens gut ein Dutzend Bücher und über 500 wissenschaftliche Publikationen.
Ein wesentliches Thema seiner berufstheoretischen Forschung und Lehre betraf die „Abwendbar gefährlichen Verläufe". So schrieb er einmal (Wien Med Wochenschr 2002;152: 257-260): „AllgemeinärztInnen müssen die ihnen zur Verfügung stehende knappe Zeitspanne unermüdlich dafür nützen, um aus abertausenden Bagatellen die so raren verborgenen abwendbar gefährlichen Verläufe herauszufiltern. Aber selbst die Gewissenhaftesten erleben einige Male im Laufe ihrer Berufstätigkeit, dass eine Verkettung unglücklicher Umstände einen abwendbar gefährlichen Verlauf unter ihren Augen unabwendbar werden lässt. Alle zur Niederlassung kommenden ÄrztInnen sollten das wissen, damit sie ohne Illusionen in ihren schweren Beruf eintreten.“

Obwohl seine Arbeiten die bestehende Ordnung in der Medizinwissenschaft durchbrachen, hat er sich doch in seinem Kampf gegen Dogmen in der Medizin viel Unverständnis und sogar Gegnerschaft eingehandelt, wurde er schließlich (von Prof. Fuchsig) zur Habilitation gedrängt. Damit war er wahrscheinlich der erste, der die Allgemeinpraxis in die Hochschule integrierte. Die Professoren Fellinger, Kraupp, Deutsch und Tuppy unterstützten ihn später und stellten ihm Lehrsäle für seine Vorlesungen zur Verfügung. Anfang der 90iger Jahre kam es aufgrund der intensiven Bemühungen von Robert Braun zur Installierung eines Instituts für Allgemeinmedizin in Wien. Die Ironie des Schicksals wollte es, dass Braun aus Altersgründen nicht mehr als Leiter fungieren konnte. Einen Meilenstein in der Angewandten Medizin stellte auch die von Braun geschaffene „Kasugraphie“, die Beschreibung für die 300 häufigsten gegeneinander abgrenzbaren Fälle von allgemeinärztlichen Beratungsproblemen dar.

An dieser Stelle soll auch seiner Gattin Margret gedankt sein, die ihm in schweren Zeiten zur Seite stand. Für sie, aber auch für alle, die Robert Braun persönlich gekannt haben, bedeutet sein Tod einen Abschied von einer großen Persönlichkeit, einem guten Freund und sicher auch für die, für die er ein offener und wohlwollender Lehrer und Mentor war.

Gerhart Hitzenberger, Wien

Der Autor möchte an dieser Stelle auch der langjährigen Mitarbeiterin Prof. Brauns, Frau Dr. Waltraud Fink, dafür danken, dass sie ihm eine Reihe biografischer Daten und Hinweise zur wissenschaftlichen Tätigkeit von Robert Braun übermittelte. 